\title{
A class of fractional integral operators with multi-index Mittag-Leffler k-function and Bessel k-function of first kind
}

\author{
Rana Safdar Ali*, Shahid Mubeen, Muhammad Mumtaz Ahmad \\ Department of Mathematics, University of Sargodha, Sargodha, Pakistan.
}

\begin{abstract}
In this paper, we discuss the multi-index Mittag Leffler k-function and Bessel k-function of the first kind in fractional calculus. We investigate fractional integral operators (Saigo's, Erdelyi Kober, Reimann Liouvill, Weyl) and extend with the product of multi-index Mittag Leffler k-function to the Bessel k-function of the first kind. Here, we establish new theorems that provide the image of multi-index Mittag Leffler and Bessel k-functions under these k-fractional operators. These results are derived in general behave and give several well-known results in the theory of multi-index k-functions.
\end{abstract}

Keywords: Fractional integral operators, generalized Mittag-Leffler k-function, Bessel k-function, classical hypergeometric functions.

2020 MSC: 45P05, 33E12, 33C10, 33C05.

(C)2021 All rights reserved.

\section{Introduction}

During century, Mittag Leffler function has significant topic in mathematics due to its numerous applications and generalization in different filed of mathematical analysis. Fractional calculus has closed relation with Mittag Leffler because of fractional problems. Hille and Tamarkin presented the solution of second kind Abel integral equation in term of Mittag Leffler function. In 1954, Barret considered the general solution of linear fractional differential equation with constant coefficient.

Recently, many researchers have great concentrate in the direction of Mittag Leffler type functions and improved a lot of results in the field of fractional calculus. Gösta Magnus Mittag Leffler discussed a power series function $E_{\alpha}(z)$ and studied its properties in five successive account ML1-ML5 at the time of $1902-$ 1905. For more detailed of Mittage Leffler type functions have immense contribution in the field of integral inequalities involved different kinds of fractional operators like as differentiable exponentially convex and quasi-convex mapping, conformal fractional integral, Grüss type integral inequalities, geometrical convex functions, generalized $(\mathrm{g}, \mathrm{s}, \mathrm{m}, \varphi)$-preinvex function, and some generalizations of Ostrowski type inequalities, we refer the interesting reader to $[2,6-9,11,15,19,23,24,32,33]$.

\footnotetext{
*Corresponding author

Email address: rsafdar0@gmail . com (Rana Safdar Ali)

doi: $10.22436 /$ jmcs.022.03.06
}

Received: 2020-03-17 Revised: 2020-04-10 Accepted: 2020-06-15 
The classical Mittag-Leffler function [14, 20] for $z \in \mathbb{C}$ and $\alpha>0$ is defined as

$$
\mathrm{E}_{\alpha}(z)=\sum_{\mathrm{n}=0}^{\infty} \frac{z^{\mathrm{n}}}{\Gamma(\alpha \mathrm{n}+1)}
$$

where $(\alpha n) !=\Gamma(\alpha n+1)$ are power terms of the exponential series. The Mittag Leffler function also called as Queen function of fractional calculus.

Wiman studied the generalization of Mittag Leffler function and defined for $\alpha, \beta>0$ and $z \in \mathbb{C}$, given as below [1]

$$
\mathrm{E}_{\alpha, \beta}(z)=\sum_{n=0}^{\infty} \frac{z^{n}}{\Gamma(\alpha \mathrm{n}+\beta)} .
$$

There are many extensions, generalization or combinations for Mittag-Leffler functions which are found in the papers [16, 27-30]. Kiryakova [18] introduced and studied the multi-index Mittag-Leffler function which is the extension of generalized Mittag-Leffler function. The multi-index Mittag-Leffler function is

$$
E_{\left(\frac{1}{\rho_{j}}, \mu_{j}\right)}(z)=\sum_{n=0}^{\infty} \frac{z^{n}}{\prod_{j=1}^{m} \Gamma\left(\mu_{j}+\frac{n}{\rho_{j}}\right)},
$$

where $m \geqslant 1, \operatorname{Re}\left(\rho_{1}\right), \ldots, \operatorname{Re}\left(\rho_{m}\right)>0$, and $\mu_{1}, \ldots, \mu_{m}$ are arbitrary parameters. If we replace $m=1$, $\rho_{1}=\frac{1}{\alpha}$ and $\mu_{1}=\beta$, then it will become the classical result which is given in equation (1.1).

Bessel functions $[4,31]$ interested from functional inequalities and geometric opinions. The first kind Bessel function of order $q$ is defined as

$$
\mathrm{J}_{\mathrm{q}}(z)=\sum_{\mathrm{n}=0}^{\infty} \frac{(-1)^{\mathrm{n}}\left(\frac{z}{2}\right)^{2 n+q}}{\Gamma(\mathrm{q}+\mathrm{n}+1) n !} .
$$

The first kind Bessel k-function [21] of order $q$ is defined for $\operatorname{Re}(q)>-1$,

$$
\mathrm{J}_{\mathrm{q} ; \mathrm{k}}(z)=\sum_{n=0}^{\infty} \frac{(-1)^{n}\left(\frac{z}{2}\right)^{2 n+\frac{q}{k}}}{\Gamma_{k}(\mathrm{q}+n k+k) n !}
$$

where $k$ is any positive real number.

Generalized fractional integral operators [17] is defined for $y>0$ and $\alpha^{\prime}, \beta^{\prime}, \eta^{\prime} \in \mathbb{C}, \operatorname{Re}\left(\alpha^{\prime}\right)>0$, as follows

$$
\left(I_{0, y}^{\alpha^{\prime}, \beta^{\prime}, \eta^{\prime}} f\right)(x)=\frac{x^{-\alpha^{\prime}-\beta^{\prime}}}{\Gamma\left(\alpha^{\prime}\right)} \int_{0}^{x}(x-t)^{\alpha^{\prime}-1}{ }_{2} F_{1}\left(\alpha^{\prime}+\beta^{\prime},-\eta^{\prime} ; \alpha^{\prime} ; 1-\frac{t}{x}\right) f(t) d t
$$

and

$$
\left(\mathrm{I}_{y, \infty}^{\alpha^{\prime}, \beta^{\prime}, \eta^{\prime}} f\right)(x)=\frac{1}{\Gamma\left(\alpha^{\prime}\right)} \int_{x}^{\infty}(\mathrm{t}-x)^{\alpha^{\prime}-1} \mathrm{t}^{-\alpha^{\prime}-\beta^{\prime}}{ }_{2} \mathrm{~F}_{1}\left(\alpha^{\prime}+\beta^{\prime},-\eta^{\prime} ; \alpha^{\prime} ; 1-\frac{x}{\mathrm{t}}\right) \mathrm{f}(\mathrm{t}) \mathrm{dt},
$$

where, Gauss hypergeometric function ${ }_{2} \mathrm{~F}_{1}$ used its kernel.

The Series of Gauss hypergeometric function [26] is defined as

$$
{ }_{2} F_{1}\left(\alpha^{\prime}, \beta^{\prime} ; \eta^{\prime} ; t\right)=\sum_{m=0}^{\infty} \frac{\left(\alpha^{\prime}\right)_{m}\left(\beta^{\prime}\right)_{m}}{\left(\eta^{\prime}\right)_{m}} \frac{t^{m}}{m !},
$$

where $\alpha^{\prime}, \beta^{\prime}, \eta^{\prime} \in \mathbb{C}, \eta^{\prime} \neq 0,-1,-2, \ldots,|t|<1\left(\alpha^{\prime}\right)_{m},\left(\beta^{\prime}\right)_{m}$ and $\left(\eta^{\prime}\right)_{m}$ are Pochhammer's symbols.

Generalized hypergeometric function [26] is defined as follows:

$$
{ }_{p} F_{q}\left(a_{1}, \ldots, a_{p} ; b_{1}, \ldots, b_{q} ; t\right)=\sum_{m=0}^{\infty} \frac{\left(a_{1}\right)_{m} \cdots\left(a_{p}\right)_{m}}{\left(b_{1}\right)_{m} \cdots\left(b_{q}\right)_{m}} \frac{t^{m}}{m !}
$$


where $a_{i}, b_{j} \in \mathbb{C}, b_{j} \neq 0,-1, \ldots(i=1,2, \ldots, p ; j=1,2, \ldots, q)$.

Pochhammer's symbol [25] is defined as

$$
(l)_{n}= \begin{cases}l(l+1)(l+2) \cdots(l+n-1), & \text { for } n \geqslant 1, \\ 1, & \text { for } n=0, l \neq 0,\end{cases}
$$

where $l \in \mathbb{C}$ and $n \in \mathbb{N}$.

Gamma function $[22,25]$ is defined for $\operatorname{Re}(u)>0$ as follows:

$$
\Gamma(z)=\int_{0}^{\infty} t^{z-1} e^{-t} d t .
$$

Beta function [25] is defined as

$$
\beta(l, h)=\int_{0}^{1} t^{l-1}(1-t)^{h-1} d t
$$

where $\operatorname{Re}(\mathrm{l})>0, \operatorname{Re}(\mathrm{h})>0$.

Gamma k-function and Beta k-function introduced by Diaz and Pariguan [5]. Fractional k-integral operators presented by Mubeen and Habibullah [22] in 2012.

Beta k-function [5] is defined as

$$
\beta_{\mathrm{k}}(l, h)=\frac{1}{k} \int_{0}^{1} s^{\frac{l}{k}-1}(1-s)^{\frac{h}{k}-1} d s, \quad \operatorname{Re}(l)>0, \quad \operatorname{Re}(h)>0 .
$$

For $k>0, \operatorname{Re}(x)$ and $\operatorname{Re}(y)$, the relation beta gamma $k$-functions can be written as

$$
\beta_{k}(x, y)=\frac{\Gamma_{k}(x) \Gamma_{k}(y)}{\Gamma_{k}(x+y)} .
$$

The integral form of the generalized gamma k-function [12] is defined as

$$
\Gamma_{k}(z)=\int_{0}^{\infty} t^{z-1} e^{\frac{-t^{k}}{k}} d t
$$

where $k \in \mathbb{R}^{+}, z \in \mathbb{C}, \operatorname{Re}(z)>0$. Some important relations about gamma function are as follows

$$
\Gamma_{\mathrm{k}}(z+\mathrm{k})=z \Gamma_{\mathrm{k}}(z), \quad \Gamma_{\mathrm{k}}(\gamma)=(\mathrm{k})^{\frac{\gamma}{k}-1} \Gamma\left(\frac{\gamma}{\mathrm{k}}\right) .
$$

The useful relation about gamma $k$-function is given as

$$
\Gamma_{k}(t+n k)=(t)_{n, k} \Gamma_{k}(t) .
$$

The generalized Mittag-Leffler k-function [10] is defined as

$$
E_{k ; v, \rho}^{\delta}(t)=\sum_{n=0}^{\infty} \frac{(\delta)_{n, k} t^{n}}{\Gamma_{k}(v n+\rho) n !},
$$

where $k \in \mathbb{R}^{+}, v, \rho, \delta, t \in \mathbb{C}, \operatorname{Re}(v)>0, \operatorname{Re}(\rho)>0$.

The hypergeometric $k$-function [13] is defined for all $\alpha^{\prime}, \beta^{\prime}, \eta^{\prime} \in \mathbb{C}$ and $\eta^{\prime} \neq 0,-1,-2,-3, \ldots,|t|<1$ as fallows:

$$
{ }_{2} \mathrm{~F}_{1, \mathrm{k}}\left(\left(\alpha^{\prime}, \mathrm{k}\right),\left(\beta^{\prime}, \mathrm{k}\right) ;\left(\eta^{\prime}, \mathrm{k}\right) ; \mathrm{t}\right)=\sum_{m=0}^{\infty} \frac{\left(\alpha^{\prime}\right)_{\mathrm{m}, \mathrm{k}}\left(\beta^{\prime}\right)_{\mathrm{m}, \mathrm{k}}}{\left(\eta^{\prime}\right)_{\mathrm{m}, \mathrm{k}}} \frac{\mathrm{t}^{\mathrm{m}}}{\mathrm{m} !}, \quad \mathrm{k}>0,
$$

where $\left(\alpha^{\prime}\right)_{m, k},\left(\beta^{\prime}\right)_{m, k}$ and $\left(\eta^{\prime}\right)_{m, k}$ are Pochhammer k-symbols. 
The generalized hypergeometric $k$-function is defined as

$$
{ }_{p} F_{q, k}\left(\left(a_{1}, k\right), \ldots,\left(a_{p}, k\right) ;\left(b_{1}, k\right), \ldots,\left(b_{q}, k\right) ; t\right)=\sum_{m=0}^{\infty} \frac{\left(a_{1}\right)_{m, k} \cdots\left(a_{p}\right)_{m, k}}{\left(b_{1}\right)_{m, k} \cdots\left(b_{q}\right)_{m, k}} \frac{t^{m}}{m !},
$$

where $a_{i}, b_{j} \in \mathbb{C}, b_{j} \neq 0,-1, \ldots(i=1,2, \ldots, p ; j=1,2, \ldots, q)$.

The Riemann Liouville fractional integral operator [3] is defined as

$$
I_{a+}^{\alpha} f(x)=\frac{1}{\Gamma(\alpha)} \int_{a}^{x}(x-t)^{\alpha-1} f(t) d t, \quad I_{b-}^{\alpha} f(x)=\frac{1}{\Gamma(\alpha)} \int_{x}^{b}(t-x)^{\alpha-1} f(t) d t,
$$

where $\operatorname{Re}(\alpha)>0$. The above integrals also called progressive or right-sided and regressive or left-sided Riemann Liouville fractional integral operators, respectively.

The left and right sided Erdelyi-Kober fractional integrals operators [3] are defined for $\alpha, \eta \in \mathbb{C}$, $\operatorname{Re}(\alpha)>0$ as follows

$$
\begin{aligned}
K_{0, u}^{\alpha, \eta} f(u) & =\frac{u^{-\alpha-\eta}}{\Gamma(\alpha)} \int_{0}^{u}(u-t)^{\alpha-1}(t)^{\eta} f(t) d t, \\
K_{u, \infty}^{\alpha, \eta} f(u) & =\frac{u^{\eta}}{\Gamma(\alpha)} \int_{u}^{\infty}(t-u)^{\alpha-1}(t)^{-\alpha-\eta} f(t) d t .
\end{aligned}
$$

The Weyl fractional integral operator [3] is defined for $\alpha \in \mathbb{C}, \operatorname{Re}(\alpha)>0$ as fallows

$$
W_{v, \infty}^{\alpha} f(v)=\frac{1}{\Gamma(\alpha)} \int_{v}^{\infty}(t-v)^{\alpha-1} f(t) d t .
$$

The Saigo's left, right type fractional $k$-integral operators are defined for $y>0$ and $\alpha^{\prime}, \beta^{\prime}, \eta^{\prime} \in \mathbb{C}$, $\operatorname{Re}\left(\alpha^{\prime}\right)>0, k \in \mathbb{R}^{+}$and Gauss hypergeometric $k$-function ${ }_{2} \mathrm{~F}_{1, \mathrm{k}}$ used its kernel, as follows

$$
\begin{aligned}
& \left(I_{k ; 0, y}^{\alpha^{\prime}, \beta^{\prime}, \eta^{\prime}} g\right)(y)=\frac{y^{\frac{-\alpha^{\prime}-\beta^{\prime}}{k}}}{k \Gamma_{k}\left(\alpha^{\prime}\right)} \int_{0}^{y}(y-s)^{\frac{\alpha^{\prime}}{k}-1}{ }_{2} F_{1, k}\left(\alpha^{\prime}+\beta^{\prime},-\eta^{\prime} ; \alpha^{\prime} ; 1-\frac{s}{y}\right) g(s) d s, \\
& \left(I_{k ; y, \infty}^{\alpha^{\prime}, \beta^{\prime}, \eta^{\prime}} g\right)(y)=\frac{1}{k \Gamma_{k}\left(\alpha^{\prime}\right)} \int_{y}^{\infty}(s-y)^{\frac{\alpha^{\prime}}{k}-1} s \frac{-\alpha^{\prime}-\beta^{\prime}}{k}{ }_{2} F_{1, k}\left(\alpha^{\prime}+\beta^{\prime},-\eta^{\prime} ; \alpha^{\prime} ; 1-\frac{y}{s}\right) g(s) d s .
\end{aligned}
$$

The Riemann Liouville fractional k-integral operators defined as follows

$$
I_{k ; a+}^{\alpha} f(x)=\frac{1}{k \Gamma_{k}(\alpha)} \int_{a}^{x}(x-t)^{\frac{\alpha}{k}-1} f(t) d t, \quad I_{k ; b-}^{\alpha} f(x)=\frac{1}{k \Gamma_{k}(\alpha)} \int_{x}^{b}(t-x)^{\frac{\alpha}{k}-1} f(t) d t,
$$

where $\operatorname{Re}(\alpha)>0$ and $k$ be any positive real number.

The Erdelyi-Kober fractional k-integral operators are defined as follows

$$
\begin{aligned}
K_{k ; 0, u}^{\alpha, \eta} f(u) & =\frac{u^{\frac{-\alpha-\eta}{k}}}{k \Gamma_{k}(\alpha)} \int_{0}^{u}(u-t)^{\frac{\alpha}{k}-1}(t)^{\frac{\eta}{k}} f(t) d t, \\
k_{k ; u, \infty}^{\alpha, \eta} f(u) & =\frac{u^{\frac{\eta}{k}}}{k \Gamma_{k}(\alpha)} \int_{u}^{\infty}(t-u)^{\frac{\alpha}{k}-1}(t)^{\frac{-\alpha-\eta}{k}} f(t) d t,
\end{aligned}
$$

where $\alpha, \eta \in \mathbb{C}, \operatorname{Re}(\alpha)>0$ and $k$ be any positive real number.

For $\alpha \in \mathbb{C}, \operatorname{Re}(\alpha)>0$ and $k \in \mathbb{R}^{+}$, the Weyl fractional $k$-integral operators are defined as

$$
W_{k ; v, \infty}^{\alpha} f(v)=\frac{1}{k \Gamma_{k}(\alpha)} \int_{v}^{\infty}(t-v)^{\frac{\alpha}{k}-1} f(t) d t .
$$

There are special cases, we generate the some relations of Bessel k-function of first kind and Mittag-Leffler k-function. 
- By Setting $j=m=1$ and $\mu_{1}=\beta, \frac{1}{\rho_{1}}=\alpha$ then, we get Wiman $k$-function,

$$
E_{k ; \alpha, \beta}(z)=\sum_{n=0}^{\infty} \frac{z^{n}}{\Gamma_{k}(\alpha n+\beta)} .
$$

- By putting $j=m=1$ and $\mu_{1}=k, \frac{1}{\rho_{1}}=\alpha$, we obtain the Mittag-Leffler $k$-function of one parameter,

$$
E_{k ; \alpha}(z)=\sum_{n=0}^{\infty} \frac{z^{n}}{\Gamma_{k}(\alpha n+k)} .
$$

- Replacing $j=m=1, \mu_{1}=\mu+k, \frac{1}{\rho_{j}}=k$, and $z=\frac{-z^{2}}{4}$ in the multiindex mittag-leffler $k$-function, we have Bessel k-function of first kind,

$$
\left(\frac{2}{z}\right)^{\frac{p}{k}} E_{k ; k, \mu+k}\left(\frac{-z^{2}}{4}\right)=J_{\mu, k}(z) .
$$

\section{Saigo's generalized fractional k-integral operators with multi-index Mittag-Leffler k-function and Bessel k-function}

In this section, we discuss composition of generalized fractional $k$-integral operators with the product of multi-index Mittag-Leffler k-function and Bessel k-function of first kind. The generalized fractional $k$-integral operators can be extended with these compositions which gave an important theory in the field of analysis. The following theorems can prove these consequences.

Theorem 2.1. Suppose that $\mathrm{m} \geqslant 1$ is an integer and $\mathrm{I}_{\mathrm{k} ; 0, z}^{\alpha, \beta, \eta}$ be the left sided Saigo's $\mathrm{k}$-integral operator and $\operatorname{Re}\left(\rho_{j}\right)>0, u_{j}(j=1, \ldots, m)$ are arbitrary parameters, then the following result holds:

$$
\begin{aligned}
& \mathrm{I}_{\mathrm{k} ; 0, z}^{\alpha, \beta, \eta}\left\{z^{\frac{\lambda}{k}-1} J_{q ; k}(z) \mathrm{E}_{k ;\left(\frac{1}{\rho_{j}}, \mu_{j}\right)}\left(a z^{\sigma}\right)\right\}=z^{\frac{\lambda-\beta+q}{k}}-1 \sum_{h=0}^{\infty} \frac{\left(\frac{1}{2}\right)^{\frac{q}{k}}\left(a z^{\sigma}\right)^{h}}{\prod_{j=1}^{m} \Gamma_{k}\left(\mu_{j}+\frac{h}{\rho_{j}}\right)} \\
& \times{ }_{2} \psi_{3}^{k}\left[\begin{array}{c|c}
(\lambda+\sigma k h+q, 2 k)(\lambda+\eta-\beta+\sigma k h+q, 2 k) \\
(q+k, k)(\lambda-\beta+\sigma k h+q, 2 k)(\lambda+\alpha+\eta+\sigma k h+q, 2 k) & \frac{-z^{2}}{4}
\end{array}\right],
\end{aligned}
$$

where $\lambda$ and $\sigma$ are arbitrary parameters and $k$ is any positive real number such that $\operatorname{Re}(\alpha)>0$ and $\alpha, \beta, \eta$ and $a$ are any complex numbers.

Proof. Consider the left sided fractional integral operators and multi-index Mittag-Leffler k-function,

$$
\begin{aligned}
& \mathrm{I}_{\mathrm{k} ; 0, z}^{\alpha, \beta, \eta}\left\{z^{\frac{\lambda}{k}-1} J_{\mathrm{q} ; \mathrm{k}}(z) \mathrm{E}_{\mathrm{k} ;\left(\frac{1}{\rho_{\mathrm{j}}}, \mu_{\mathrm{j}}\right)}\left(\mathrm{a} z^{\sigma}\right)\right\} \\
& =\frac{(z)^{\frac{-\alpha-\beta}{k}}}{k \Gamma_{k}(\alpha)} \int_{0}^{z}(z-t)^{\frac{\alpha}{k}-1}{ }_{2} F_{1, k}\left(\alpha+\beta,-\eta ; \alpha ;\left(1-\frac{t}{z}\right)\right) \sum_{p, h=0}^{\infty} \frac{t^{\frac{\lambda}{k}-1}(-1)^{p}\left(\frac{t}{2}\right)^{2 p+\frac{q}{k}}\left(a t^{\sigma}\right)^{h}}{\Gamma_{k}(q+p k+k) p ! \prod_{j=1}^{m} \Gamma_{k}\left(\mu_{j}+\frac{h}{\rho_{j}}\right)} d t \\
& =\frac{z^{\frac{-\alpha-\beta}{k}}}{k \Gamma_{k}(\alpha)} \int_{0}^{z} \frac{(z-t)^{\frac{\alpha}{k}-1}}{(t)^{1-\frac{\lambda}{k}}} \sum_{n=0}^{\infty} \frac{(\alpha+\beta)_{n, k}(-\eta)_{n, k}\left(1-\frac{t}{z}\right)^{n}}{(\alpha)_{n, k} n !} \sum_{p, h=0}^{\infty} \frac{(-1)^{p}\left(\frac{t}{2}\right)^{2 p+\frac{q}{k}}\left(a(t)^{\sigma}\right)^{h}}{\Gamma_{k}(q+p k+k) p ! \prod_{j=1}^{m} \Gamma_{k}\left(\mu_{j}+\frac{h}{\rho_{j}}\right)} d t \\
& =\frac{z^{\frac{-\alpha-\beta}{k}}}{k \Gamma_{k}(\alpha)} \sum_{n=0}^{\infty} \frac{(\alpha+\beta)_{n}(-\eta)_{n, k}}{(\alpha)_{n, k} n !} \sum_{p, h=0}^{\infty} \frac{(-1)^{p}\left(\frac{1}{2}\right)^{2 p+\frac{q}{k}} a^{h}}{\Gamma_{k}(q+p k+k) p ! \prod_{j=1}^{m} \Gamma_{k}\left(\mu_{j}+\frac{h}{\rho_{j}}\right)} \int_{0}^{z} \frac{(z-t)^{\frac{\alpha}{k}-1}\left(1-\frac{t}{z}\right)^{n}}{t^{1-\frac{\lambda+\sigma h k+2 p k+q}{k}}} d t \\
& =\frac{z^{\frac{-\beta}{k}-1}}{k \Gamma_{k}(\alpha)} \sum_{n=0}^{\infty} \frac{(\alpha+\beta)_{n, k}(-\eta)_{n, k}}{(\alpha)_{n, k} n !} \xi_{p, h} \int_{0}^{z}\left(1-\frac{t}{z}\right)^{\frac{\alpha}{k}+n-1} t^{\frac{\lambda+\sigma h k+2 p k+q}{k}}-1 d t \text {, }
\end{aligned}
$$


where

$$
\xi_{p, h}=\sum_{p, h=0}^{\infty} \frac{(-1)^{p}\left(\frac{1}{2}\right)^{2 p+\frac{q}{k}} a^{h}}{\Gamma_{k}(q+p k+k) p ! \prod_{j=1}^{m} \Gamma_{k}\left(\mu_{j}+\frac{h}{\rho_{j}}\right)} .
$$

By putting these values $\frac{\mathrm{t}}{z}=\mathrm{u} \quad \Rightarrow \mathrm{t}=\mathrm{uz} \quad \Rightarrow \mathrm{dt}=z \mathrm{du} \quad \mathrm{t}=0 \Rightarrow \mathrm{u}=0 \quad \mathrm{t}=z \quad \Rightarrow \mathrm{u}=1$ in the equation (2.1), we obtain

$$
\begin{aligned}
& \mathrm{I}_{\mathrm{k} ; 0, z}^{\alpha, \beta, \eta}\left\{z^{\frac{\lambda}{k}-1} J_{\mathrm{q} ; \mathrm{k}}(z) \mathrm{E}_{\mathrm{k} ;\left(\frac{1}{\rho_{j}}, \mu_{j}\right)}\left(\mathrm{a} z^{\sigma}\right)\right\} \\
& =\frac{z^{\frac{-\beta}{k}}-1}{k \Gamma_{k}(\alpha)} \sum_{n=0}^{\infty} \frac{(\alpha+\beta)_{n, k}(-\eta)_{n, k}}{(\alpha)_{n, k} n !} \xi_{p, h} \int_{0}^{1}(1-u)^{\frac{\alpha}{k}+n-1}(u z)^{\frac{\lambda+\sigma h k+2 p k+q}{k}-1} z d u \\
& =\frac{z^{\frac{\lambda-\beta}{k}-1}}{k \Gamma_{k}(\alpha)} \sum_{n=0}^{\infty} \frac{(\alpha+\beta)_{n, k}(-\eta)_{n, k}}{(\alpha)_{n, k} n !} \xi_{p, h} z^{2 p+\sigma h+\frac{q}{k}} \int_{0}^{1}(1-u)^{\frac{\alpha}{k}+n-1} u^{\frac{\lambda+\sigma h k+2 p k+q}{k}-1} d u .
\end{aligned}
$$

By using equation (1.2) in the equation (2.2), we get

$$
\begin{aligned}
\mathrm{I}_{k ; 0, z}^{\alpha, \beta, \eta}\left\{z^{\frac{\lambda}{k}-1} J_{q ; k}(z) E_{k ;\left(\frac{1}{\rho_{j}}, \mu_{j}\right)}\left(a z^{\sigma}\right)\right\} \\
\quad=\frac{z^{\frac{\lambda-\beta}{k}}-1}{\Gamma_{k}(\alpha)} \sum_{n=0}^{\infty} \frac{(\alpha+\beta)_{n, k}(-\eta)_{n, k}}{(\alpha)_{n, k} n !} \xi_{p, h} z^{2 p+\sigma h+\frac{q}{k}} \beta_{k}(\lambda+\sigma h k+2 p k+q, \alpha+k n) .
\end{aligned}
$$

By using equation (1.3) in the equation (2.3), we have

$$
\begin{aligned}
\mathrm{I}_{k ; 0, z}^{\alpha, \beta, \eta}\left\{z^{\frac{\lambda}{k}-1} J_{q ; k}(z) E_{k ;\left(\frac{1}{\rho_{j}}, \mu_{j}\right)}\left(a z^{\sigma}\right)\right\} \\
\quad=\frac{z^{\frac{\lambda-\beta}{k}}-1}{\Gamma_{k}(\alpha)} \sum_{n=0}^{\infty} \frac{(\alpha+\beta)_{n, k}(-\eta)_{n, k}}{(\alpha)_{n, k} n !} \xi_{p, h} z^{2 p+\sigma h+\frac{q}{k}} \frac{\Gamma_{k}(\lambda+\sigma h k+2 p k+q) \Gamma_{k}(\alpha+k n)}{\Gamma_{k}(\lambda+\sigma h k+2 p k+q+\alpha+k n)} .
\end{aligned}
$$

By using the equation (1.4) in the equation (2.4), we obtain

$$
\begin{aligned}
\mathrm{I}_{k ; 0, z}^{\alpha, \beta, \eta}\{ & \left\{z^{\frac{\lambda}{k}-1} J_{q ; k}(z) E_{k ;\left(\frac{1}{\rho_{j}}, \mu_{j}\right)}\left(a z^{\sigma}\right)\right\} \\
& =\frac{z^{\frac{\lambda-\beta}{k}}-1}{\Gamma_{k}(\alpha)} \sum_{n=0}^{\infty} \frac{(\alpha+\beta)_{n, k}(-\eta)_{n, k}}{(\alpha)_{n, k} n !} \xi_{p, h} \frac{z^{2 p+\sigma h+\frac{q}{k}} \Gamma_{k}(\lambda+\sigma h k+2 p k+q)(\alpha)_{n, k} \Gamma_{k}(\alpha)}{\Gamma_{k}(\lambda+\sigma h k+2 p k+q+\alpha)(\lambda+\sigma h k+2 p k+q+\alpha)_{n, k}} \\
& =z^{\frac{\lambda-\beta}{k}-1} \xi_{p, h} z^{2 p+\sigma h+\frac{q}{k}} \sum_{n=0}^{\infty} \frac{(\alpha+\beta)_{n, k}(-\eta)_{n, k}(1)^{n} \Gamma_{k}(\lambda+\sigma h k+2 p k+q)}{(n !) \Gamma_{k}(\lambda+\sigma h k+2 p k+q+\alpha)(\lambda+\sigma h k+2 p k+q+\alpha)_{n, k}} .
\end{aligned}
$$

Since

$$
{ }_{2} \mathrm{~F}_{1, k}(\mathrm{a}, \mathrm{b} ; \mathrm{c} ; 1)=\frac{\Gamma_{k}(c) \Gamma_{k}(c-a-b)}{\Gamma_{k}(c-a) \Gamma_{k}(c-b)} .
$$

Therefore, the equation (2.5), becomes

$$
\begin{aligned}
& \mathrm{I}_{k ; 0, z}^{\alpha, \beta, \eta}\left\{z^{\frac{\lambda}{k}-1} J_{q ; k}(z) E_{k ;\left(\frac{1}{\rho_{j}}, \mu_{j}\right)}\left(a z^{\sigma}\right)\right\} \\
&=\sum_{p, h=0}^{\infty} \frac{z^{\frac{\lambda-\beta}{k}-1}(-1)^{p}\left(\frac{z}{2}\right)^{2 p+\frac{q}{k}}\left(a z^{\sigma}\right)^{h}}{\Gamma_{k}(q+p k+k) p ! \prod_{j=1}^{m} \Gamma_{k}\left(\mu_{j}+\frac{h}{\rho_{j}}\right)} \\
& \times \frac{\Gamma_{k}(\lambda+\sigma k h+2 p k+q) \Gamma_{k}(\lambda+\eta-\beta+\sigma k h+2 p k+q)}{\Gamma_{k}(\lambda-\beta+\sigma k h+2 p k+q) \Gamma_{k}(\lambda+\alpha+\eta+\sigma k h+2 p k+q)} \\
&= \sum_{h=0}^{\infty} \frac{z^{\frac{\lambda-\beta+q}{k}-1}\left(\frac{1}{2}\right)^{\frac{q}{k}}\left(a z^{\sigma}\right)^{h}}{\prod_{j=1}^{m} \Gamma_{k}\left(\mu_{j}+\frac{h}{\rho_{j}}\right)} 2 \psi_{3}^{k}\left[\begin{array}{c}
(\lambda+\sigma k h+q, 2 k)(\lambda+\eta-\beta+\sigma k h+q, 2 k) \\
(q+k, k)(\lambda-\beta+\sigma k h+q, 2 k)(\lambda+\alpha+\eta+\sigma k h+q, 2 k)
\end{array}\right] .
\end{aligned}
$$


Corollary 2.2. Suppose that $\mathrm{m} \geqslant 1$ is an integer and $\mathrm{I}_{\mathrm{k} ; 0, z}^{\alpha, \beta, \eta}$ be the left sided Saigo's $k$-integral operator and $\operatorname{Re}\left(\rho_{j}\right)>0, u_{j}(j=1, \ldots, m)$ are arbitrary parameters, then the following result holds:

$$
\begin{aligned}
& \mathrm{I}_{\mathrm{k} ; 0, z}^{\alpha, \beta, \eta}\left\{z^{\frac{\lambda}{k}-1} \mathrm{~J}_{\mathrm{q} ; \mathrm{k}}(z) \mathrm{E}_{\mathrm{k} ;\left(\frac{1}{\rho_{j}}, \mu_{j}\right)}\left(\mathrm{a} z^{\sigma}\right)\right\}=z^{\frac{\lambda-\beta}{k}-1} \sum_{\mathrm{p}=0}^{\infty} \frac{(-1)^{\mathrm{p}}\left(\frac{z}{2}\right)^{2 p+\frac{\mathrm{q}}{k}}}{\Gamma_{\mathrm{k}}(\mathrm{q}+\mathrm{pk}+\mathrm{k}) \mathrm{p} !} \\
& \times{ }_{3} \psi_{3}^{k}\left[\begin{array}{c|c}
(\lambda+q+2 k p, \sigma k)(\lambda+\eta-\beta+q+2 k p, \sigma k)(k, k) \\
\left.(\lambda-\beta+q+2 k p, \sigma k)(\lambda+\alpha+\eta+q+2 k p, \sigma k)\left(\mu_{j}, \frac{1}{\rho_{j}}\right)\right|_{j=1} ^{m} & \frac{a z^{\sigma}}{k}
\end{array}\right],
\end{aligned}
$$

where $\lambda$ and $\sigma$ are arbitrary parameters and $\mathrm{k}$ is any positive real number such that $\operatorname{Re}(\alpha)>0$ and $\alpha, \beta, \eta$ and $\mathrm{a}$ are any complex numbers.

Corollary 2.3. Left sided Saigo's k-integral operator, where $\operatorname{Re}(\alpha)>0$ and $\alpha, \beta, \eta$ and $\sigma$ are any complex numbers then the following result holds:

$$
\begin{aligned}
& \mathrm{I}_{k ; 0, z}^{\alpha, \beta, \eta}\left\{J_{p, k}(z) J_{q, k}\left(z^{\sigma}\right)\right\}=z^{\frac{q \sigma-\beta-k}{k}} \sum_{n=0}^{\infty} \frac{(-1)^{n}\left(\frac{z}{2}\right)^{2 n+\frac{p}{k}\left(\frac{1}{2}\right)^{\frac{\sigma q}{k}}}}{n ! \Gamma_{k}(p+n k+k)} \\
& \quad \times{ }_{2} \Psi_{3}^{k}\left[\begin{array}{c}
(2 n k+p+k+q \sigma k, 2 \sigma)(\eta-\beta+2 n k+p+k+q \sigma k, 2 \sigma) \\
(2 n k+p+k+q \sigma k-\beta, 2 \sigma)(\alpha+\eta 2 n k+p+k+q \sigma k, 2 \sigma)(q+k, k)
\end{array} \mid\left(\frac{-z}{2}\right)^{2 \sigma}\right] .
\end{aligned}
$$

Theorem 2.4. Suppose that $\mathrm{m} \geqslant 1$ and $\mathrm{I}_{k ; z, \infty}^{\alpha, \beta, \eta}$ be the right sided Saigo's $k$-integral operator and $\operatorname{Re}\left(\rho_{j}\right)>0$, $\mathrm{u}_{\mathfrak{j}}(\mathfrak{j}=1, \ldots, \mathrm{m})$ are arbitrary parameters then this result holds:

$$
\begin{aligned}
& \mathrm{I}_{\mathrm{k} ; z, \infty}^{\alpha, \beta, \eta}\left\{z^{\frac{\lambda}{k}} J_{q ; k}(z) E_{k ;\left(\frac{1}{\rho_{j}}, \mu_{j}\right)} \mathrm{a} z^{-\sigma}\right\}=z^{\frac{\lambda-\beta+q}{k}} \sum_{h=0}^{\infty} \frac{\left(\frac{1}{2}\right)^{\frac{q}{k}}\left(a z^{-\sigma}\right)^{h}}{\prod_{j=1}^{m} \Gamma_{k}\left(\mu_{j}+\frac{h}{\rho_{j}}\right)} \\
& \quad \times{ }_{2} \psi_{3}^{k}\left[\begin{array}{c}
(\beta-\lambda+\sigma k h-q,-2 k)(\eta-\lambda+\sigma k h-q,-2 k) \\
(q+k, k)(-\lambda+\sigma k h-q,-2 k)(\alpha+\beta+\eta-\lambda+\sigma k h-q,-2 k)
\end{array}\right.
\end{aligned}
$$

where $\lambda$ and $\sigma$ are arbitrary parameters and $k \in \mathbb{R}^{+}$such that $\operatorname{Re}(\alpha)>0$ and $\alpha, \beta, \eta$ and a are any complex numbers.

Proof. Consider the right sided fractional integral operator and multi-index Mittag-Leffler k-function

$$
\begin{aligned}
& \mathrm{I}_{\mathrm{k} ; z, \infty}^{\alpha, \beta, \eta^{\prime}}\left\{z^{\frac{\lambda}{k}} J_{q ; k}(z) \mathrm{E}_{k ;\left(\frac{1}{\rho_{j}}, \mu_{j}\right)} \mathrm{a} z^{-\sigma}\right\} \\
& =\frac{1}{k \Gamma_{k}(\alpha)} \int_{z}^{\infty} \frac{(t-z)^{\frac{\alpha}{k}-1}}{t^{\frac{\alpha+\beta}{k}}}{ }_{2} F_{1, k}\left(\alpha+\beta,-\eta ; \alpha ;\left(1-\frac{z}{t}\right)\right) t^{\frac{\lambda}{k}} \sum_{p, h=0}^{\infty} \frac{(-1)^{p}\left(\frac{t}{2}\right)^{2 p+\frac{q}{k}}\left(a t^{-\sigma}\right)^{h}}{\Gamma_{k}(q+p k+k) p ! \prod_{j=1}^{m} \Gamma_{k}\left(\mu_{j}+\frac{h}{\rho_{j}}\right)} d t \\
& =\frac{1}{k \Gamma_{k}(\alpha)} \int_{z}^{\infty} \frac{(t-z)^{\frac{\alpha}{k}-1}}{t^{\frac{\alpha+\beta}{k}}} \sum_{n=0}^{\infty} \frac{(\alpha+\beta)_{n, k}(-\eta)_{n, k}}{(\alpha)_{n, k} n !}\left(1-\frac{z}{t}\right)^{n} \sum_{p, h=0}^{\infty} \frac{t^{\frac{\lambda}{k}}(-1)^{p}\left(\frac{t}{2}\right)^{2 p+\frac{q}{k}}\left(a t^{-\sigma}\right)^{h}}{\Gamma_{k}(q+p k+k) p ! \prod_{j=1}^{m} \Gamma_{k}\left(\mu_{j}+\frac{h}{\rho_{j}}\right)} d t \\
& =\frac{1}{k \Gamma_{k}(\alpha)} \sum_{n=0}^{\infty} \frac{(\alpha+\beta)_{n, k}(-\eta)_{n, k}}{(\alpha)_{n, k} n !} \sum_{p, h=0}^{\infty} \frac{(-1)^{p}\left(\frac{1}{2}\right)^{2 p+\frac{q}{k} a^{h}}}{\Gamma_{k}(q+p k+k) p ! \prod_{j=1}^{m} \Gamma_{k}\left(\mu_{j}+\frac{h}{\rho_{j}}\right)} \int_{z}^{\infty} \frac{(t-z)^{\frac{\alpha}{k}-1}\left(1-\frac{z}{t}\right)^{n}}{t^{\frac{-\lambda+\alpha+\beta+\sigma h k-2 p k-q}{k}}} d t \\
& =\frac{1}{k \Gamma_{k}(\alpha)} \sum_{n=0}^{\infty} \frac{(\alpha+\beta)_{n, k}(-\eta)_{n, k}}{(\alpha)_{n, k} n !} \xi_{p, h} \int_{z}^{\infty}\left(1-\frac{z}{t}\right)^{\frac{\alpha}{k}+n-1} t^{\frac{\lambda-\beta-\sigma h k+2 p k+q}{k}-1} d t \text {, }
\end{aligned}
$$

where

$$
\xi_{p, h}=\sum_{p, h=0}^{\infty} \frac{(-1)^{p}\left(\frac{1}{2}\right)^{2 p+\frac{q}{k}} a^{h}}{\Gamma_{k}(q+p k+k) p ! \prod_{j=1}^{m} \Gamma_{k}\left(\mu_{j}+\frac{h}{\rho_{j}}\right)} .
$$


By putting the values $\frac{z}{t}=u \Rightarrow t=\frac{z}{u}, t=z \Rightarrow u=1, t=\infty \Rightarrow u=0, \frac{z}{t}=u \Rightarrow d t=\frac{-t^{2}}{z} d u, d t=$ $-\frac{z}{u^{2}} d u$ in the equation (2.6), we obtain

$$
\begin{aligned}
& \mathrm{I}_{k ; z, \infty}^{\alpha, \beta, \eta_{i}}\left\{z^{\frac{\lambda}{k}} J_{q ; k}(z) E_{k ;\left(\frac{1}{\rho_{j}}, \mu_{j}\right)} a z^{-\sigma}\right\} \\
& =\frac{1}{k \Gamma_{k}(\alpha)} \sum_{n=0}^{\infty} \frac{(\alpha+\beta)_{n, k}(-\eta)_{n, k}}{(\alpha)_{n, k} n !} \xi_{p, h} \int_{1}^{0}(1-u)^{\frac{\alpha}{k}+n-1}\left(\frac{z}{u}\right)^{\frac{\lambda-\beta-\sigma h k+2 p k+q}{k}}-1\left(\frac{-z}{u^{2}}\right) d u \\
& =\frac{1}{k \Gamma_{k}(\alpha)} \sum_{n=0}^{\infty} \frac{(\alpha+\beta)_{n, k}(-\eta)_{n, k}}{(\alpha)_{n, k} n !} \xi_{p, h} \int_{0}^{1}(1-u)^{\frac{\alpha}{k}+n-1} z^{\frac{\lambda-\beta}{k}} z^{-\sigma h} z^{2 p+\frac{q}{k}} u^{\frac{-\lambda+\beta+\sigma h k-2 p k-q}{k}}-1 d u \\
& =\frac{z^{\frac{\lambda-\beta}{k}}}{\Gamma_{k}(\alpha)} \sum_{n=0}^{\infty} \frac{(\alpha+\beta)_{n, k}(-\eta)_{n, k}}{(\alpha)_{n, k} n !} \xi_{p, h} z^{2 p-\sigma h+\frac{q}{k}} \frac{1}{k} \int_{0}^{1}(1-u)^{\frac{\alpha}{k}+n-1} u^{\frac{-\lambda+\beta+\sigma h k-2 p k-q}{k}-1} d u .
\end{aligned}
$$

By using the equation (1.2) in the equation (2.7), we get

$$
\begin{aligned}
& \mathrm{I}_{\mathrm{k} ; z, \infty}^{\alpha, \beta, \eta_{i}}\left\{z^{\frac{\lambda}{k}} J_{q ; k}(z) E_{k ;\left(\frac{1}{\left.\rho_{j}, \mu_{j}\right)}\right.} \mathrm{a} z^{-\sigma}\right\} \\
& \quad=\frac{z^{\frac{\lambda-\beta}{k}}}{\Gamma_{k}(\alpha)} \sum_{n=0}^{\infty} \frac{(\alpha+\beta)_{n, k}(-\eta)_{n, k}}{(\alpha)_{n, k} n !} \xi_{p, h} z^{2 p-\sigma h+\frac{q}{k}} \beta_{k}(-\lambda+\beta+\sigma h k-2 p k-q, \alpha+k n) .
\end{aligned}
$$

By using the equation (1.3) in the equation (2.8), we have

$$
\begin{aligned}
& \mathrm{I}_{k ; z, \infty}^{\alpha, \beta, \eta_{i}}\left\{z^{\frac{\lambda}{k}} J_{q ; k}(z) E_{k ;\left(\frac{1}{\rho_{j}}, \mu_{j}\right)} a z^{-\sigma}\right\} \\
& =\frac{z^{\frac{\lambda-\beta}{k}}}{\Gamma_{k}(\alpha)} \sum_{n=0}^{\infty} \frac{(\alpha+\beta)_{n, k}(-\eta)_{n, k}}{(\alpha)_{n, k} n !} \xi_{p, h} z^{2 p-\sigma h+\frac{q}{k}} \frac{\Gamma_{k}(\beta-\lambda+\sigma k h-2 p k-q) \Gamma_{k}(\alpha+k n)}{\Gamma_{k}(\beta-\lambda+\sigma k h-2 p k-q+\alpha+k n)} .
\end{aligned}
$$

Since

$$
\Gamma_{\mathrm{k}}(\alpha+\mathrm{kn})=(\alpha)_{\mathrm{n}, \mathrm{k}} \Gamma_{\mathrm{k}}(\alpha) .
$$

Therefore, using the equation (2.9) in equation (2.10), we obtain

$$
\begin{aligned}
& \mathrm{I}_{k ; z, \infty}^{\alpha, \beta, \eta}\left\{z^{\frac{\lambda}{k}} J_{q ; k}(z) E_{k ;\left(\frac{1}{\rho_{j}}, \mu_{j}\right)} a z^{-\sigma}\right\} \\
& =z^{\frac{\lambda-\beta}{k}} \xi_{p, h} \sum_{n=0}^{\infty} \frac{z^{2 p-\sigma h+\frac{q}{k}}(\alpha+\beta)_{n, k}(-\eta)_{n, k} \Gamma_{k}(\beta-\lambda+\sigma k h-2 p k-q)(\alpha)_{n, k} \Gamma_{k}(\alpha)}{\Gamma_{k}(\alpha)(\alpha)_{n, k} n !(\beta-\lambda+\sigma k h-2 p k-q+\alpha)_{n, k} \Gamma_{k}(\sigma k h-2 p k-q+\alpha+\beta-\lambda)} \\
& =z^{\frac{\lambda-\beta}{k}} \xi_{p, h} z^{2 p-\sigma h+\frac{q}{k}} \sum_{n=0}^{\infty} \frac{(\alpha+\beta)_{n, k}(-\eta)_{n, k}(1)^{n}}{n !(\beta-\lambda+\sigma k h-2 p k-q+\alpha)_{n, k}} \frac{\Gamma_{k}(\beta-\lambda+\sigma k h-2 p k-q)}{\Gamma_{k}(\sigma k h-2 p k-q+\alpha+\beta-\lambda)} .
\end{aligned}
$$

Since

$$
{ }_{2} \mathrm{~F}_{1, k}(\mathrm{a}, \mathrm{b} ; \mathrm{c} ; 1)=\frac{\Gamma_{\mathrm{k}}(\mathrm{c}) \Gamma_{\mathrm{k}}(\mathrm{c}-\mathrm{a}-\mathrm{b})}{\Gamma_{\mathrm{k}}(\mathrm{c}-\mathrm{a}) \Gamma_{\mathrm{k}}(\mathrm{c}-\mathrm{b})} .
$$

Therefore, the equation (2.11), becomes

$$
\begin{aligned}
& \mathrm{I}_{k ; z, \infty}^{\alpha, \beta, \eta_{i}}\left\{z^{\frac{\lambda}{k}} J_{q ; k}(z) E_{k ;\left(\frac{1}{\rho_{j}}, \mu_{j}\right)} a z^{-\sigma}\right\} \\
& =\sum_{p, h=0}^{\infty} \frac{z^{\frac{\lambda-\beta}{k}}(-1)^{p}\left(\frac{z}{2}\right)^{2 p+\frac{q}{k}}\left(a z^{-\sigma}\right)^{h}}{\Gamma_{k}(q+p k+k) p ! \prod_{j=1}^{m} \Gamma_{k}\left(\mu_{j}+\frac{h}{\rho_{j}}\right)} \\
& \quad \times \frac{\Gamma_{k}(\beta-\lambda+\sigma k h-2 p k-q) \Gamma_{k}(\eta-\lambda+\sigma k h-2 p k-q)}{\Gamma_{k}(-\lambda+\sigma k h-2 p k-q) \Gamma_{k}(\alpha+\beta+\eta-\lambda+\sigma k h-2 p k-q)}
\end{aligned}
$$




$$
=\sum_{h=0}^{\infty} \frac{\left(\frac{1}{2}\right)^{\frac{q}{k}} z^{\frac{\lambda-\beta+q}{k}}\left(a z^{-\sigma}\right)^{h}}{\prod_{j=1}^{m} \Gamma_{k}\left(\mu_{j}+\frac{h}{\rho_{j}}\right)} 2 \psi_{3}^{k}\left[\begin{array}{c|c}
(\beta-\lambda+\sigma k h-q,-2 k)(\eta-\lambda+\sigma k h-q,-2 k) \\
(q+k, k)(-\lambda+\sigma k h-q,-2 k)(\alpha+\beta+\eta-\lambda+\sigma k h-q,-2 k)
\end{array} \mid \frac{-z^{2}}{4}\right] .
$$

Corollary 2.5. Suppose that $\mathrm{m} \geqslant 1$ and $\mathrm{I}_{\mathrm{k} ; z, \infty}^{\alpha, \beta, \eta}$ be the right sided Saigo's k-integral operator and $\operatorname{Re}\left(\rho_{j}\right)>0$, $u_{\mathfrak{j}}(j=1, \ldots, m)$ are arbitrary parameters then this result holds:

$$
\begin{aligned}
& \mathrm{I}_{\mathrm{k} ; z, \infty}^{\alpha, \beta, \eta}\left\{z^{\frac{\lambda}{k}} J_{\mathrm{q} ; \mathrm{k}}(z) \mathrm{E}_{\mathrm{k} ;\left(\frac{1}{\rho_{j}}, \mu_{j}\right)} \mathrm{a} z^{-\sigma}\right\}=z^{\frac{\lambda-\beta}{k}} \sum_{p=0}^{\infty} \frac{(-1)^{p}\left(\frac{z}{2}\right)^{2 p+\frac{q}{k}}}{\Gamma_{k}(q+p k+k) p !} \\
& \times{ }_{3} \psi_{3}^{k}\left[\begin{array}{c|c}
(\beta-\lambda-q-2 k p, \sigma k)(\eta-\lambda-q-2 k p, \sigma k)(k, k) & a z^{-\sigma} \\
\left.(-\lambda-q-2 k p, \sigma k)(\alpha+\beta+\eta-\lambda-q-2 k p, \sigma k)\left(\mu_{j}, \frac{1}{\rho_{j}}\right)\right|_{j=1} ^{m} & k
\end{array}\right],
\end{aligned}
$$

where $\lambda$ and $\sigma$ are arbitrary parameters and $k \in \mathbb{R}^{+}$such that $\operatorname{Re}(\alpha)>0$ and $\alpha, \beta, \eta$ and a are any complex numbers.

Corollary 2.6. The right sided Saigo's k-integral operator for $m \geqslant 1$ and $\operatorname{Re}\left(\rho_{j}\right)>0, u_{j}(j=1, \ldots, m)$ are arbitrary parameters then this result holds:

$$
\begin{aligned}
\mathrm{I}_{k ; z, \infty}^{\alpha, \beta, \eta}\left\{\mathrm{E}_{\mathrm{k} ;\left(\frac{1}{\rho_{j}}, \mu_{j}\right)}(z) \mathrm{E}_{\mathrm{k} ;\left(\frac{1}{\rho_{j}}, \mu_{j}\right)} \mathrm{a} z^{\sigma}\right\}= & z^{\frac{-\beta}{k}} \sum_{n=0}^{\infty} \frac{\left(a z^{\sigma}\right)^{n}}{\prod_{j=1}^{m} \Gamma_{k}\left(\mu_{j}+\frac{n}{\rho_{j}}\right)} \\
& \times{ }_{3} \Psi_{3}^{k}\left[\begin{array}{c}
(\beta-\sigma n k,-k)(\eta-\sigma n k,-k)(k, k) \\
\left.(-n k,-k)(\alpha+\beta+\eta-\sigma n k,-k)\left(u_{j}, \frac{1}{\rho_{j}}\right)\right|_{j=1} ^{m} \mid \frac{z}{k}
\end{array}\right],
\end{aligned}
$$

where $\lambda$ and $\sigma$ are arbitrary parameters and $\mathrm{k} \in \mathbb{R}^{+}$such that $\operatorname{Re}(\alpha)>0$ and $\alpha, \beta, \eta$ are complex numbers.

\section{Erdelyi-Kober fractional k-integral operator with multi-index Mittag-Leffler k-function and Bessel k-function}

In this section, we solve Erdelyi Kobber fractional k-integrals with the product of multi-index MittagLeffler and Bessel $k$-functions in the form of theorems.

Theorem 3.1. Suppose that $\mathrm{m} \geqslant 1$ is an integer and $\mathrm{K}_{\mathrm{k} ; 0, z}^{\alpha, \eta}$ be the Erdelyi-Kober integral operator and $\operatorname{Re}\left(\rho_{j}\right)>0$, $u_{\mathfrak{j}}(j=1, \ldots, m)$ are arbitrary parameters, then this result holds:

$$
\begin{aligned}
& K_{k ; 0, z}^{\alpha, \eta}\left\{z^{\frac{\lambda}{k}-1} J_{q ; k}(z) E_{k ;\left(\frac{1}{\rho_{j}}, \mu_{j}\right)} a z^{\sigma}\right\} \\
& \quad=z^{\frac{\lambda+q}{k}-1} \sum_{h=0}^{\infty} \frac{\left(\frac{1}{2}\right)^{\frac{q}{k}}\left(a z^{\sigma}\right)^{h}}{\prod_{j=1}^{m} \Gamma_{k}\left(\mu_{j}+\frac{h}{\rho_{j 5}}\right)} 1 \psi_{2}^{k}\left[\begin{array}{c}
(\lambda+\eta+\sigma k h+q, 2 k) \\
(q+k, k)(\lambda+\alpha+\eta+\sigma k h+q, 2 k)
\end{array} \mid \frac{-z^{2}}{4}\right],
\end{aligned}
$$

where $\eta, \alpha$ and $\mathrm{a}$ are any complex numbers and $\mathrm{k} \in \mathbb{R}^{+}$.

Proof. Consider the Erdelyi-kober fractional k-integral and multi-index Mittag-Leffler k-function:

$$
\begin{aligned}
& \mathrm{K}_{\mathrm{k} ; 0, z}^{\alpha, \eta}\left\{z^{\frac{\lambda}{k}-1} J_{q ; k}(z) E_{k ;\left(\frac{1}{\rho_{j}}, \mu_{j}\right)} a z^{\sigma}\right\} \\
& =\frac{z^{\frac{-\alpha-\eta}{k}}}{k \Gamma_{k}(\alpha)} \int_{0}^{z}(z-t)^{\frac{\alpha}{k}-1} t^{\frac{\eta}{k}} t^{\frac{\lambda}{k}}-1 \sum_{p, h=0}^{\infty} \frac{(-1)^{p}\left(\frac{t}{2}\right)^{2 p+\frac{q}{k}}\left(a t^{\sigma}\right)^{h}}{\Gamma_{k}(q+p k+k) p ! \prod_{j=1}^{m} \Gamma_{k}\left(\mu_{j}+\frac{h}{\rho_{j}}\right)} d t \\
& =\frac{z^{\frac{-\alpha-\eta}{k}}}{k \Gamma_{k}(\alpha)} \sum_{p, h=0}^{\infty} \frac{(-1)^{p}\left(\frac{1}{2}\right)^{2 p+\frac{q}{k}} a^{h}}{\Gamma_{k}(q+p k+k) p ! \prod_{j=1}^{m} \Gamma_{k}\left(\mu_{j}+\frac{h}{\rho_{j}}\right)} \int_{0}^{z}(z-t)^{\frac{\alpha}{k}-1} t^{\frac{\lambda+\eta+\sigma h k+2 p k+q}{k}}-1 d t \\
& =\frac{z^{\frac{-\eta}{k}-1}}{k \Gamma_{k}(\alpha)} \xi_{p, h} \int_{0}^{z}\left(1-\frac{t}{z}\right)^{\frac{\alpha}{k}-1} t^{\frac{\lambda+\eta+\sigma h k+2 p k+q}{k}-1} d t \text {, }
\end{aligned}
$$


where

$$
\xi_{p, h}=\sum_{p, h=0}^{\infty} \frac{(-1)^{p}\left(\frac{1}{2}\right)^{2 p+\frac{q}{k}} a^{h}}{\Gamma_{k}(q+p k+k) p ! \prod_{j=1}^{m} \Gamma_{k}\left(\mu_{j}+\frac{h}{\rho_{j}}\right)}
$$

By putting these values $\frac{t}{z}=u \Rightarrow t=u z \Rightarrow d t=z d u \quad t=0 \Rightarrow u=0 \quad t=z \Rightarrow u=1$ in the equation (3.1), we obtain

$$
\begin{aligned}
& \mathrm{K}_{\mathrm{k} ; 0, z}^{\alpha, \eta}\left\{z^{\frac{\lambda}{\mathrm{k}}-1} \mathrm{~J}_{\mathrm{q} ; \mathrm{k}}(z) \mathrm{E}_{\mathrm{k} ;\left(\frac{1}{\rho_{j}}, \mu_{j}\right)} \mathrm{a} z^{\sigma}\right\}=\frac{z^{\frac{-\eta}{k}-1}}{\mathrm{k} \Gamma_{k}(\alpha)} \xi_{p, h} \int_{0}^{1}(1-u)^{\frac{\alpha}{k}-1}(u z)^{\frac{\lambda+\eta+\sigma h k+2 p k+q}{k}}-1 z d u \\
& =\frac{z^{\frac{\lambda}{k}-1}}{k \Gamma_{k}(\alpha)} \xi_{p, h} z^{2 p+\sigma h+\frac{q}{k}} \int_{0}^{1}(1-u)^{\frac{\alpha}{k}-1} u^{\frac{\lambda+\eta+\sigma h k+2 p k+q}{k}}-1 d u .
\end{aligned}
$$

By using the equation (1.2) in the equation (3.2), we get

$$
\mathrm{K}_{\mathrm{k} ; 0, z}^{\alpha, \eta}\left\{z^{\frac{\lambda}{k}-1} J_{q ; k}(z) \mathrm{E}_{k ;\left(\frac{1}{\rho_{j}}, \mu_{j}\right)} \mathrm{a} z^{\sigma}\right\}=\frac{z^{\frac{\lambda}{k}-1}}{\Gamma_{k}(\alpha)} \xi_{p, h} z^{2 p+\sigma h+\frac{q}{k}} \beta_{k}(\eta+\lambda+\sigma k h+2 p k+q, \alpha) .
$$

By using the equation (1.3) in the equation (3.3), we have

$$
\begin{aligned}
& \mathrm{K}_{\mathrm{k} ; 0, z}^{\alpha, \eta}\left\{z^{\frac{\lambda}{k}-1} J_{q ; k} E_{k ;\left(\frac{1}{\rho_{j}}, \mu_{j}\right)} a z^{\sigma}\right\} \\
& =\frac{z^{\frac{\lambda}{k}-1}}{\Gamma_{k}(\alpha)} \xi_{p, h} z^{2 p+\sigma h+\frac{q}{k}} \frac{\Gamma_{k}(\lambda+\eta+\sigma k h+2 p k+q) \Gamma_{k}(\alpha)}{\Gamma_{k}(\alpha+\lambda+\eta+\sigma k h+2 p k+q)} \\
& =z^{\frac{\lambda}{k}-1} \sum_{p, h=0}^{\infty} \frac{(-1)^{p}\left(\frac{z}{2}\right)^{2 p+\frac{q}{k}}\left(a z^{\sigma}\right)^{h}}{\Gamma_{k}(q+p k+k) p ! \prod_{j=1}^{m} \Gamma_{k}\left(\mu_{j}+\frac{h}{\rho_{j}}\right)} \frac{\Gamma_{k}(\lambda+\eta+\sigma k h+2 p k+q)}{\Gamma_{k}(\alpha+\lambda+\eta+\sigma k h+2 p k+q)} \\
& =z^{\frac{\lambda+q}{k}}-1 \sum_{h=0}^{\infty} \frac{\left(\frac{1}{2}\right)^{\frac{q}{k}}\left(a z^{\sigma}\right)^{h}}{\prod_{j=1}^{m} \Gamma_{k}\left(\mu_{j}+\frac{h}{\rho_{j 5}}\right)} 1 \psi_{2}^{k}\left[\begin{array}{c|c}
(\lambda+\eta+\sigma k h+q, 2 k) \\
(q+k, k)(\lambda+\alpha+\eta+\sigma k h+q, 2 k)
\end{array} \mid \frac{-z^{2}}{4}\right] .
\end{aligned}
$$

Corollary 3.2. Suppose that $\mathrm{m} \geqslant 1$ is an integer and $\mathrm{K}_{\mathrm{k} ; 0, z}^{\alpha, \eta}$ be the Erdelyi-Kober integral operator and $\operatorname{Re}\left(\rho_{j}\right)>0$, $u_{\mathfrak{j}}(j=1, \ldots, m)$ are arbitrary parameters, then this result holds:

$$
\begin{aligned}
& \mathrm{K}_{\mathrm{k} ; 0, z}^{\alpha, \eta}\left\{z^{\frac{\lambda}{k}-1} J_{\mathrm{q} ; \mathrm{k}}(z) \mathrm{E}_{\mathrm{k} ;\left(\frac{1}{\rho_{j}}, \mu_{j}\right)} \mathrm{a} z^{\sigma}\right\} \\
& \quad=z^{\frac{\lambda}{k}-1} \sum_{p=0}^{\infty} \frac{(-1)^{p}\left(\frac{z}{2}\right)^{2 p+\frac{q}{k}}}{\Gamma_{k}(q+p k+k) p !} 2 \psi_{2}^{k}\left[\begin{array}{c}
(\lambda+\eta+q+2 k p, \sigma k)(k, k) \\
\left.(\lambda+\alpha+\eta+q+2 k p, \sigma k)\left(\mu_{j}, \frac{1}{\rho_{j}}\right)\right|_{j=1} ^{m}
\end{array} \mid \frac{a z^{\sigma}}{k}\right],
\end{aligned}
$$

where $\eta, \alpha$ and a are any complex numbers and $k \in \mathbb{R}^{+}$.

Theorem 3.3. Suppose that $\mathrm{m} \geqslant 1$ and $\mathrm{K}_{\mathrm{k}_{; z, \infty}, \mathrm{n}}$ be the Erdelyi-Kober fractional $\mathrm{k}$-integral operator and $\operatorname{Re}\left(\rho_{\mathrm{j}}\right)>0$, $u_{\mathfrak{j}}(j=1, \ldots, m)$ are arbitrary parameters then this result holds:

$$
\begin{aligned}
& \mathrm{K}_{\mathrm{k} ; z, \infty}^{\alpha, \eta}\left\{z^{\frac{\lambda}{k}} J_{\mathrm{q} ; \mathrm{k}}(z) \mathrm{E}_{\mathrm{k} ;\left(\frac{1}{\rho_{j}}, \mu_{j}\right)} \mathrm{a} z^{-\sigma}\right\} \\
& =z^{\frac{\lambda+q}{k}} \sum_{h=0}^{\infty} \frac{\left(\frac{1}{2}\right)^{\frac{q}{k}}\left(a z^{-\sigma}\right)^{h}}{\prod_{j=1}^{m} \Gamma_{k}\left(\mu_{j}+\frac{h}{\rho_{j}}\right)} 1 \psi_{2}^{k}\left[\begin{array}{c|c}
(\eta-\lambda+\sigma k h-q,-2 k) \\
(q+k, k)(\alpha+\eta-\lambda+\sigma k h-q .-2 k)
\end{array} \mid \frac{-z^{2}}{4}\right],
\end{aligned}
$$

where $\alpha, \eta$, a are complex numbers, $\mathrm{k}$ is any positive real number and $\operatorname{Re}(\alpha)>0$. 
Proof. Consider Erdelyi-Kober fractional k-integral and multi-index Miitag-Leffler k-function

$$
\begin{aligned}
& \mathrm{K}_{\mathrm{k} ; z, \infty}^{\alpha, \eta}\left\{z^{\frac{\lambda}{k}} J_{q ; k}(z) \mathrm{E}_{\mathrm{k} ;\left(\frac{1}{\rho_{j}}, \mu_{j}\right)} \mathrm{a} z^{-\sigma}\right\} \\
& =\frac{z^{\frac{\eta}{k}}}{k \Gamma_{k}(\alpha)} \int_{z}^{\infty}(\mathrm{t}-z)^{\frac{\alpha}{k}-1} \mathrm{t}^{\frac{-\alpha-\eta}{k}} \mathrm{t}^{\frac{\lambda}{k}} \sum_{p, h=0}^{\infty} \frac{(-1)^{p}\left(\frac{\mathrm{t}}{2}\right)^{2 p+\frac{q}{k}}\left(a t^{-\sigma}\right)^{h}}{\Gamma_{k}(\mathrm{q}+p k+k) p ! \prod_{j=1}^{m} \Gamma_{k}\left(\mu_{j}+\frac{h}{\rho_{j}}\right)} d t \\
& =\frac{z^{\frac{\eta}{k}}}{k \Gamma_{k}(\alpha)} \sum_{p, h=0}^{\infty} \frac{(-1)^{p}\left(\frac{1}{2}\right)^{2 p+\frac{q}{k}} a^{h}}{\Gamma_{k}(q+p k+k) p ! \prod_{j=1}^{m} \Gamma_{k}\left(\mu_{j}+\frac{h}{\rho_{j}}\right)} \int_{z}^{\infty}(t-z)^{\frac{\alpha}{k}-1} t^{\frac{\lambda-\alpha-\eta}{k}} t^{-\sigma h+2 p+\frac{q}{k}} d t \\
& =\frac{z^{\frac{\eta}{k}}}{k \Gamma_{k}(\alpha)} \xi_{p, h} \int_{z}^{\infty}\left(1-\frac{z}{t}\right)^{\frac{\alpha}{k}-1} t^{\frac{\lambda-\eta-\sigma h k+2 p k+q}{k}}-1 d t,
\end{aligned}
$$

where

$$
\xi_{p, h}=\sum_{p, h=0}^{\infty} \frac{(-1)^{p}\left(\frac{1}{2}\right)^{2 p+\frac{q}{k}} a^{h}}{\Gamma_{k}(q+p k+k) p ! \prod_{j=1}^{m} \Gamma_{k}\left(\mu_{j}+\frac{h}{\rho_{j}}\right)} .
$$

By substituting $\frac{z}{\mathrm{t}}=\mathrm{u} \Rightarrow \mathrm{t}=\frac{z}{\mathfrak{u}} \mathrm{t}=z \Rightarrow \mathrm{u}=1 \mathrm{t}=\infty \Rightarrow \mathrm{u}=0 \frac{\mathrm{z}}{\mathrm{t}}=\mathrm{u} \Rightarrow \mathrm{dt}=\frac{-\mathrm{t}^{2}}{\mathrm{z}} \mathrm{du} \quad \mathrm{dt}=-\frac{z}{\mathfrak{u}^{2}} \mathrm{du}$ in the equation (3.4), we obtain

$$
\begin{aligned}
\mathrm{K}_{\mathrm{k} ; z, \infty}^{\alpha, n}\left\{z^{\frac{\lambda}{k}} J_{q ; k} E_{k ;\left(\frac{1}{\rho_{j}}, \mu_{j}\right)} a z^{-\sigma}\right\} & =\frac{z^{\frac{\eta}{k}}}{k \Gamma_{k}(\alpha)} \xi_{p, h} \int_{1}^{0}(1-u)^{\frac{\alpha}{k}-1}\left(\frac{z}{u}\right)^{\frac{\lambda-\eta-\sigma h k+2 p k+q}{k}-1}\left(-\frac{z}{u^{2}}\right) d u \\
& =\frac{z^{\frac{\eta}{k}}}{k \Gamma_{k}(\alpha)} \xi_{p, h} \int_{0}^{1}(1-u)^{\frac{\alpha}{k}-1} z^{\frac{\lambda-\eta-\sigma h+2 p k+q}{k}} u^{\frac{-\lambda+\eta+\sigma h k-2 p k-q}{k}-1} d u \\
& =\frac{z^{\frac{\lambda}{k}}}{\Gamma_{k}(\alpha)} \xi_{p, h} z^{2 p-\sigma h+\frac{q}{k}} \frac{1}{k} \int_{0}^{1}(1-u)^{\frac{\alpha}{k}-1} u^{\frac{-\lambda+\eta+\sigma h k-2 p k-q}{k}-1} d u .
\end{aligned}
$$

By using the equation (1.2) in the equation (3.5), we get

$$
\mathrm{K}_{k ; z, \infty}^{\alpha, n}\left\{z^{\frac{\lambda}{k}} J_{q ; k}(z) E_{k ;\left(\frac{1}{\rho_{j}}, \mu_{j}\right)} a z^{-\sigma}\right\}=\frac{z^{\frac{\lambda}{k}}}{\Gamma_{k}(\alpha)} \xi_{p, h} z^{2 p-\sigma h+\frac{q}{k}} \beta_{k}(\eta-\lambda+\sigma k h-2 p k-q, \alpha) .
$$

By using the equation (1.3) in the equation (3.6), we have

$$
\begin{aligned}
& \mathrm{K}_{\mathrm{k} ; z, \infty}^{\alpha, n}\left\{z^{\frac{\lambda}{k}} J_{\mathrm{q} ; \mathrm{k}}(z) \mathrm{E}_{\mathrm{k} ;\left(\frac{1}{\rho_{j}}, \mu_{j}\right)} \mathrm{a} z^{-\sigma}\right\} \\
& =\frac{z^{\frac{\lambda}{k}}}{\Gamma_{k}(\alpha)} \xi_{p, h} z^{2 p-\sigma h+\frac{\mathrm{q}}{k}} \frac{\Gamma_{k}(\eta-\lambda+\sigma k h-2 p k-q) \Gamma_{k}(\alpha)}{\Gamma_{k}(\eta-\lambda+\sigma k h-2 p k-q+\alpha)} \\
& =z^{\frac{\lambda}{k}} \sum_{p, h=0}^{\infty} \frac{(-1)^{p}\left(\frac{z}{2}\right)^{2 p+\frac{q}{k}}\left(a z^{-\sigma}\right)^{h}}{\Gamma_{k}(q+p k+k) p ! \prod_{j=1}^{m} \Gamma_{k}\left(\mu_{j}+\frac{h}{\rho_{j}}\right)} \frac{\Gamma_{k}(\eta-\lambda+\sigma k h-2 p k-q)}{\Gamma_{k}(\eta-\lambda+\sigma k h-2 p k-q+\alpha)} \\
& =z^{\frac{\lambda+q}{k}} \sum_{h=0}^{\infty} \frac{\left(\frac{1}{2}\right)^{\frac{q}{k}}\left(a z^{-\sigma}\right)^{h}}{\prod_{j=1}^{m} \Gamma_{k}\left(\mu_{j}+\frac{h}{\rho_{j}}\right)} 1 \psi_{2}^{k}\left[\begin{array}{c}
(\eta-\lambda+\sigma k h-q,-2 k) \\
(q+k, k)(\alpha+\eta-\lambda+\sigma k h-q .-2 k)
\end{array} \mid \frac{-z^{2}}{4}\right] .
\end{aligned}
$$

Corollary 3.4. Suppose that $\mathrm{m} \geqslant 1$ and $\mathrm{K}_{\mathrm{k} ; z, \infty}^{\alpha, \eta}$ be the Erdelyi-Kober fractional $\mathrm{k}$-integral operator and $\operatorname{Re}\left(\rho_{\mathrm{j}}\right)>0$, $u_{j}(j=1, \ldots, m)$ are arbitrary parameters then this result holds:

$$
\begin{aligned}
& \mathrm{K}_{\mathrm{k} ; z, \infty}^{\alpha, n}\left\{z^{\frac{\lambda}{k}} J_{q ; k}(z) E_{k ;\left(\frac{1}{\rho_{j}}, \mu_{j}\right)} a z^{-\sigma}\right\} \\
& =z^{\frac{\lambda}{k}} \sum_{p=0}^{\infty} \frac{(-1)^{p}\left(\frac{z}{2}\right)^{2 p+\frac{q}{k}}}{\Gamma_{k}(q+p k+k) p !} 2 \psi_{2}^{k}\left[\begin{array}{c|c}
(\eta-\lambda-q-2 k p, \sigma k)(k, k) \\
\left.(\alpha+\eta-\lambda-q-2 k p, \sigma k)\left(\mu_{j}, \frac{1}{\rho_{j}}\right)\right|_{j=1} ^{m}
\end{array} \mid \frac{a z^{-\sigma}}{k}\right],
\end{aligned}
$$

where $\alpha, \eta$, a are complex numbers, $\mathrm{k}$ is any positive real number and $\operatorname{Re}(\alpha)>0$. 


\section{Riemann-Liouville fractional k-integral operator and multi-index Mittag-Leffler k-function and Bessel k-function}

In this section, we solved the results in which elaborated the relation between the multi-index MittagLeffler k-function with Bessel k-function and Riemann-Liouville fractional k-integral operator.

Theorem 4.1. Suppose that $\mathrm{m} \geqslant 1$ is an integer and $\mathrm{R}_{0, z}^{\alpha}$ be the Riemann-Liouville fractional $\mathrm{k}$-integral operator and $\operatorname{Re}\left(\rho_{j}\right)>0, u_{j}(j=1, \ldots, m)$ are arbitrary parameters then this result holds:

$$
\begin{aligned}
& \mathrm{R}_{\mathrm{k} ; 0, z}^{\alpha}\left\{z^{\frac{\lambda}{k}-1} J_{\mathrm{q} ; \mathrm{k}}(z) \mathrm{E}_{\mathrm{k} ;\left(\frac{1}{\rho_{j}}, \mu_{j}\right)} \mathrm{a} z^{\sigma}\right\} \\
& \quad=z^{\frac{\lambda+\alpha+q}{k}}-1 \sum_{h=0}^{\infty} \frac{\left(\frac{1}{2}\right)^{\frac{\mathrm{q}}{k}}\left(\mathrm{a} z^{\sigma}\right)^{h}}{\prod_{j=1}^{m} \Gamma_{k}\left(\mu_{j}+\frac{h}{\rho_{j}}\right)} 1 \psi_{2}^{k}\left[\begin{array}{c}
(\lambda+\sigma k h+q, 2 k) \\
(\mathrm{q}+\mathrm{k}, \mathrm{k})(\lambda+\alpha+\sigma k h+q, 2 k)
\end{array} \mid \frac{-z^{2}}{4}\right],
\end{aligned}
$$

where $\alpha$, a are complex numbers, $k \in \mathbb{R}^{+}$and $\operatorname{Re}(\alpha)>0$.

Proof. Consider Riemann-Liouville fractional k-integral and multi-index Mittag-Leffler kfunction

$$
\begin{aligned}
& R_{k ; 0, z}^{\alpha}\left\{z^{\frac{\lambda}{k}-1} J_{q ; k}(z) E_{k ;\left(\frac{1}{\rho_{j}}, \mu_{j}\right)} a z^{\sigma}\right\} \\
& =\frac{1}{k \Gamma_{k}(\alpha)} \int_{0}^{z}(z-t)^{\frac{\alpha}{k}-1} t^{\frac{\lambda}{k}-1} \sum_{p, h=0}^{\infty} \frac{(-1)^{p}\left(\frac{t}{2}\right)^{2 p+\frac{q}{k}}\left(a t^{\sigma}\right)^{h}}{\Gamma_{k}(q+p k+k) p ! \prod_{j=1}^{m} \Gamma_{k}\left(\mu_{j}+\frac{h}{\rho_{j}}\right)} d t \\
& =\frac{1}{k \Gamma_{k}(\alpha)} \sum_{p, h=0}^{\infty} \frac{(-1)^{p}\left(\frac{1}{2}\right)^{2 p+\frac{q}{k}} a^{h}}{\Gamma_{k}(q+p k+k) p ! \prod_{j=1}^{m} \Gamma_{k}\left(\mu_{j}+\frac{h}{\rho_{j}}\right)} \int_{0}^{z}(z-t)^{\frac{\alpha}{k}-1} t^{\frac{\lambda}{k}-1} t^{\sigma h+2 p+\frac{q}{k}} d t \\
& =\frac{1}{k \Gamma_{k}(\alpha)} \xi_{p, h} \int_{0}^{z} z^{\frac{\alpha}{k}-1}\left(1-\frac{t}{z}\right)^{\frac{\alpha}{k}-1} t^{\frac{\lambda}{k}-1+\sigma h+2 p+\frac{q}{k}} d t \text {, }
\end{aligned}
$$

where

$$
\xi_{p, h}=\sum_{p, h=0}^{\infty} \frac{(-1)^{p}\left(\frac{1}{2}\right)^{2 p+\frac{q}{k}} a^{h}}{\Gamma_{k}(q+p k+k) p ! \prod_{j=1}^{m} \Gamma_{k}\left(\mu_{j}+\frac{h}{\rho_{j}}\right)} .
$$

By putting these values $\frac{t}{z}=u \quad \Rightarrow t=u z \Rightarrow d t=z d u \quad t=0 \Rightarrow u=0, t=z \quad \Rightarrow u=1$ in the equation (4.1), we get

$$
\begin{aligned}
& \mathrm{R}_{\mathrm{k} ; 0, z}^{\alpha}\left\{z^{\frac{\lambda}{k}-1} J_{\mathrm{q} ; \mathrm{k}}(z) \mathrm{E}_{\mathrm{k} ;\left(\frac{1}{\rho_{j}}, \mu_{\mathrm{j}}\right)} \mathrm{a} z^{\sigma}\right\}=\frac{1}{\mathrm{k} \Gamma_{\mathrm{k}}(\alpha)} \xi_{\mathrm{p}, \mathrm{h}} \int_{0}^{1} z^{\frac{\alpha}{k}-1}(1-\mathrm{u})^{\frac{\alpha}{k}-1}(z \mathrm{u})^{\frac{\lambda+q}{k}-1+\sigma h+2 p} z \mathrm{du} \\
& =\frac{1}{k \Gamma_{k}(\alpha)} \xi_{p, h} \int_{0}^{1} z^{\frac{\alpha+\lambda+q}{k}-1+\sigma h+2 p}(1-u)^{\frac{\alpha}{k}-1} u^{\frac{\lambda+q}{k}-1+\sigma h+2 p} d u \\
& =\frac{z^{\frac{\alpha+\lambda}{k}-1}}{\Gamma_{k}(\alpha)} \xi_{p, h} z^{2 p+\sigma h+\frac{q}{k}} \frac{1}{k} \int_{0}^{1}(1-u)^{\frac{\alpha}{k}-1} u^{\frac{\lambda+q+\sigma k h+2 p k}{k}}-1 d u
\end{aligned}
$$

By using the equation (1.2) in the equation (4.2), we have

$$
\mathrm{R}_{\mathrm{k} ; 0, z}^{\alpha}\left\{z^{\frac{\lambda}{k}-1} J_{\mathrm{q} ; \mathrm{k}}(z) \mathrm{E}_{\mathrm{k} ;\left(\frac{1}{\rho_{j}}, \mu_{j}\right)} a z^{\sigma}\right\}=\frac{z^{\frac{\alpha+\lambda}{k}-1}}{\Gamma_{k}(\alpha)} \xi_{p, h} z^{2 p+\sigma h+\frac{q}{k}} \beta_{k}(\lambda+\sigma k h+2 p k+q, \alpha) .
$$

By using the equation (1.3) in the equation (4.3), we obtain

$$
\mathrm{R}_{\mathrm{k} ; 0, z}^{\alpha}\left\{z^{\frac{\lambda}{k}-1} J_{q ; k}(z) E_{k ;\left(\frac{1}{\rho_{j}}, \mu_{j}\right)} a z^{\sigma}\right\}
$$




$$
\begin{aligned}
& =\frac{z^{\frac{\alpha+\lambda}{k}-1}}{\Gamma_{k}(\alpha)} \xi_{p, h} z^{2 p+\sigma h+\frac{q}{k}} \frac{\Gamma_{k}(\lambda+\sigma k h+2 p k+q) \Gamma_{k}(\alpha)}{\Gamma_{k}(\lambda+\sigma k h+2 p k+q+\alpha)} \\
& =z^{\frac{\alpha+\lambda}{k}-1} \sum_{p, h=0}^{\infty} \frac{(-1)^{p}\left(\frac{z}{2}\right)^{2 p+\frac{q}{k}}\left(a z^{\sigma}\right)^{h}}{\Gamma_{k}(q+p k+k) p ! \prod_{j=1}^{m} \Gamma_{k}\left(\mu_{j}+\frac{h}{\rho_{j}}\right)} \frac{\Gamma_{k}(\lambda+\sigma k h+2 p k+q)}{\Gamma_{k}(\lambda+\sigma k h+2 p k+q+\alpha)} \\
& =z^{\frac{\lambda+\alpha+q}{k}}-1 \sum_{h=0}^{\infty} \frac{\left(\frac{1}{2}\right)^{\frac{q}{k}}\left(a z^{\sigma}\right)^{h}}{\prod_{j=1}^{m} \Gamma_{k}\left(\mu_{j}+\frac{h}{\rho_{j}}\right)} 1 \psi_{2}^{k}\left[\begin{array}{c}
(\lambda+\sigma k h+q, 2 k) \\
(q+k, k)(\lambda+\alpha+\sigma k h+q, 2 k)
\end{array} \mid \frac{-z^{2}}{4}\right] .
\end{aligned}
$$

Corollary 4.2. Suppose that $\mathrm{m} \geqslant 1$ is an integer and $\mathrm{R}_{0, z}^{\alpha}$ be the Riemann-Liouville fractional $\mathrm{k}$-integral operator and $\operatorname{Re}\left(\rho_{j}\right)>0, u_{j}(j=1, \ldots, m)$ are arbitrary parameters then this result holds:

$$
\begin{aligned}
& \mathrm{R}_{\mathrm{k} ; 0, z}^{\alpha}\left\{z^{\frac{\lambda}{k}-1} J_{q ; k}(z) \mathrm{E}_{k ;\left(\frac{1}{\rho_{j}}, \mu_{j}\right)} \mathrm{a} z^{\sigma}\right\} \\
& \quad=z^{\frac{\lambda+\alpha}{k}-1} \sum_{p=0}^{\infty} \frac{(-1)^{p}\left(\frac{z}{2}\right)^{2 p+\frac{q}{k}}}{\Gamma_{k}(q+p k+k) p !} 2 \psi_{2}^{k}\left[\begin{array}{c}
(\lambda+q+2 k p, \sigma k)(k, k) \\
\left.(\lambda+\alpha+q+2 k p, \sigma k)\left(\mu_{j}, \frac{1}{\rho_{j}}\right)\right|_{j=1} ^{m}
\end{array} \mid \frac{a z^{\sigma}}{k}\right],
\end{aligned}
$$

where $\alpha$, a are complex numbers, $k \in \mathbb{R}^{+}$and $\operatorname{Re}(\alpha)>0$.

Corollary 4.3. The Riemann-Liouville fractional k-integral operator and $\operatorname{Re}(\mu)>0, \operatorname{Re}(v)>0$ and $k \in \mathbb{R}^{+}$, $\operatorname{Re}(\mathbf{q})>-1$ then this result holds:

$$
R_{k ; 0, z}^{\alpha}\left\{J_{q ; k}\left(z^{\nu k}\right) E_{k ; \mu, v} z^{q / k}\right\}=z^{\frac{\alpha}{k}-1} \sum_{n=0}^{\infty} \frac{(-1)^{n}\left(\frac{z^{2 k}}{2}\right)^{2 n+\frac{q}{k}}}{n ! \Gamma_{k}(q+n k+k)} 2_{1}^{k}\left[\begin{array}{c}
(k(2 n v k+q v+1), q)(k, k) \\
(\alpha+k(2 n v k+q v+1), q)
\end{array} \mid \frac{z^{q / k}}{k}\right] .
$$

\section{Weyl fractional k-integral operator with multi-index Mittag-Leffler k-function Bessel k-function}

In this section, we discussed product of multi-index Mittag-Leffler and Bessel k-functions with Weyl fractional k-integral operator in the form of theorem.

Theorem 5.1. Suppose that $\mathrm{m} \geqslant 1$ is an integer and $\mathrm{W}_{\mathrm{k} ; z, \infty}^{\alpha, \eta}$ be the Weyl fractional $\mathrm{k}$-integral operator and $\operatorname{Re}\left(\rho_{j}\right)>0, u_{j}(j=1, \ldots, m)$ are arbitrary parameters then this result holds:

$$
\begin{aligned}
& W_{k ; z, \infty}^{\alpha}\left\{z^{\frac{\lambda}{k}} J_{q ; k}(z) E_{k ;\left(\frac{1}{\rho_{j}}, \mu_{j}\right)}\left(a z^{-\sigma}\right)\right\} \\
& \quad=z^{\frac{\alpha+\lambda+q}{k}} \sum_{h=0}^{\infty} \frac{\left(\frac{1}{2}\right)^{\frac{q}{k}}\left(a z^{-\sigma}\right)^{h}}{\prod_{j=1}^{m} \Gamma_{k}\left(\mu_{j}+\frac{h}{\rho_{j}}\right)} 1 \psi_{2}^{k}\left[\begin{array}{c}
(-\alpha-\lambda+\sigma k h-q,-2 k) \\
(q+k, k)(\sigma k h-q-\lambda,-2 k)
\end{array} \mid \frac{-z^{2}}{4}\right],
\end{aligned}
$$

where $\alpha$ and $\mathrm{a}$ be any complex number, $\mathrm{k} \in \mathbb{R}^{+}$.

Proof. Consider Weyl fractional k-integrals and multi-index Mittag-leffler k-function

$$
\begin{aligned}
& W_{k ; z, \infty}^{\alpha}\left\{z^{\frac{\lambda}{k}} J_{q ; k}(z) E_{k ;\left(\frac{1}{\rho_{j}}, \mu_{j}\right)}\left(a z^{-\sigma}\right)\right\} \\
& =\frac{1}{k \Gamma_{k}(\alpha)} \int_{z}^{\infty}(t-z)^{\frac{\alpha}{k}-1} t^{\frac{\lambda}{k}} \sum_{p, h=0}^{\infty} \frac{(-1)^{p}\left(\frac{t}{2}\right)^{2 p+\frac{q}{k}}\left(a t^{-\sigma}\right)^{h}}{\Gamma_{k}(q+p k+k) p ! \prod_{j=1}^{m} \Gamma_{k}\left(\mu_{j}+\frac{h}{\rho_{j}}\right)} d t \\
& =\frac{1}{k \Gamma_{k}(\alpha)} \sum_{p, h=0}^{\infty} \frac{(-1)^{p}\left(\frac{1}{2}\right)^{2 p+\frac{q}{k}} a^{h}}{\Gamma_{k}(q+p k+k) p ! \prod_{j=1}^{m} \Gamma_{k}\left(\mu_{j}+\frac{h}{\rho_{j}}\right)} \int_{z}^{\infty}(t-z)^{\frac{\alpha}{k}-1} t^{\frac{\lambda}{k}} t^{-\sigma h+2 p+\frac{q}{k}} d t \\
& =\frac{1}{k \Gamma_{k}(\alpha)} \xi_{p, h} \int_{z}^{\infty}\left(1-\frac{z}{t}\right)^{\frac{\alpha}{k}-1} t^{\frac{\lambda+\alpha-\sigma h k+2 p k+q}{k}-1} d t,
\end{aligned}
$$


where

$$
\xi_{p, h}=\sum_{p, h=0}^{\infty} \frac{(-1)^{p}\left(\frac{1}{2}\right)^{2 p+\frac{q}{k}} a^{h}}{\Gamma_{k}(q+p k+k) p ! \prod_{j=1}^{m} \Gamma_{k}\left(\mu_{j}+\frac{h}{\rho_{j}}\right)} .
$$

By substituting $\frac{z}{t}=u \Rightarrow d t=\frac{-t^{2}}{z} d u d t=-\frac{z}{u^{2}} d u \frac{z}{t}=u \Rightarrow t=\frac{z}{u} \quad t=z \Rightarrow u=1 \quad t=\infty \Rightarrow u=0$ in the equation (5.1), we have

$$
\begin{aligned}
W_{k ; z, \infty}^{\alpha}\left\{z^{\frac{\lambda}{k}} J_{q ; k}(z) E_{k ;\left(\frac{1}{\rho_{j}}, \mu_{j}\right)}\left(a z^{-\sigma}\right)\right\} & =\frac{1}{k \Gamma_{k}(\alpha)} \xi_{p, h} \int_{1}^{0}(1-u)^{\frac{\alpha}{k}-1}\left(\frac{z}{u}\right)^{\frac{\lambda+\alpha-\sigma h k+2 p k+q}{k}-1}\left(-\frac{z}{u^{2}}\right) d u \\
& =\frac{z^{\frac{\alpha+\lambda}{k}}}{\Gamma_{k}(\alpha)} \xi_{p, h} z^{2 p-\sigma h+\frac{q}{k}} \frac{1}{k} \int_{0}^{1}(1-u)^{\frac{\alpha}{k}-1} u^{\frac{-\alpha-\lambda+\sigma h k-2 p k-q}{k}}-1 d u .
\end{aligned}
$$

By using the equation (1.2) in the equation (5.2), we get

$$
W_{k ; z, \infty}^{\alpha}\left\{z^{\frac{\lambda}{k}} J_{q ; k}(z) E_{k ;\left(\frac{1}{\rho_{j}}, \mu_{j}\right)}\left(a z^{-\sigma}\right)\right\}=\frac{z^{\frac{\alpha+\lambda}{k}}}{\Gamma_{k}(\alpha)} \xi_{p, h} z^{2 p-\sigma h+\frac{q}{k}} \beta_{k}(-\alpha-\lambda+\sigma k h-2 p k-q, \alpha) .
$$

Therefore, the equation (5.3), becomes

$$
\begin{aligned}
& W_{k ; z, \infty}^{\alpha}\left\{z^{\frac{\lambda}{k}} J_{q ; k}(z) E_{k ;\left(\frac{1}{\rho_{j}}, \mu_{j}\right)}\left(a z^{-\sigma}\right)\right\} \\
& =\frac{z^{\frac{\alpha+\lambda}{k}}}{\Gamma_{k}(\alpha)} \xi_{p, h} z^{2 p-\sigma h+\frac{q}{k}} \frac{\Gamma_{k}(-\alpha-\lambda+\sigma k h-2 p k-q) \Gamma_{k}(\alpha)}{\Gamma_{k}(-\alpha-\lambda+\sigma k h-2 p k-q+\alpha)} \\
& =z^{\frac{\alpha+\lambda}{k}} \sum_{p, h=0}^{\infty} \frac{(-1)^{p}\left(\frac{z}{2}\right)^{2 p+\frac{q}{k}}\left(a z^{-\sigma}\right)^{h}}{\Gamma_{k}(q+p k+k) p ! \prod_{j=1}^{m} \Gamma_{k}\left(\mu_{j}+\frac{h}{\rho_{j}}\right)} \frac{\Gamma_{k}(-\alpha-\lambda+\sigma h k-2 p k-q)}{\Gamma_{k}(\sigma k h-2 p k-q-\lambda)} \\
& =z^{\frac{\alpha+\lambda+q}{k}} \sum_{h=0}^{\infty} \frac{\left(\frac{1}{2}\right)^{\frac{q}{k}}\left(a z^{-\sigma}\right)^{h}}{\prod_{j=1}^{m} \Gamma_{k}\left(\mu_{j}+\frac{h}{\rho_{j}}\right)} 1 \psi_{2}^{k}\left[\begin{array}{c}
(-\alpha-\lambda+\sigma k h-q,-2 k) \\
(q+k, k)(\sigma k h-q-\lambda,-2 k)
\end{array} \mid \frac{-z^{2}}{4}\right] .
\end{aligned}
$$

Corollary 5.2. Suppose that $\mathrm{m} \geqslant 1$ is an integer and $\mathrm{W}_{\mathrm{k} ; z, \infty}^{\alpha, \eta}$ be the Weyl fractional $\mathrm{k}$-integral operator and $\operatorname{Re}\left(\rho_{j}\right)>0, u_{j}(j=1, \ldots, m)$ are arbitrary parameters then this result holds:

$$
\begin{aligned}
& W_{k ; z, \infty}^{\alpha}\left\{z^{\frac{\lambda}{k}} J_{q ; k}(z) E_{k ;\left(\frac{1}{\rho_{j}}, \mu_{j}\right)}\left(a z^{-\sigma}\right)\right\} \\
& \quad=z^{\frac{\alpha+\lambda}{k}} \sum_{p=0}^{\infty} \frac{(-1)^{p}\left(\frac{z}{2}\right)^{2 p+\frac{q}{k}}}{\Gamma_{k}(q+p k+k) p !} 2 \psi_{2}^{k}\left[\begin{array}{c}
(-\alpha-\lambda-q-2 k p, \sigma k)(k, k) \\
\left.(-q-\lambda-2 k p, \sigma k)\left(\mu_{j}, \frac{1}{\rho_{j}}\right)\right|_{j=1} ^{m}
\end{array} \mid \frac{a z^{-\sigma}}{k}\right],
\end{aligned}
$$

where $\alpha$ and $\mathrm{a}$ be any complex number, $\mathrm{k} \in \mathbb{R}^{+}$.

Corollary 5.3. Suppose the Weyl fractional k-integral operator for $\operatorname{Re}(\beta)>0, \operatorname{Re}(\alpha)>0$ and $\mathrm{q}, \beta$ are arbitrary complex numbers and $\mathrm{k} \in \mathbb{R}^{+}$then this result holds:

$$
W_{k ; z, \infty}^{\alpha}\left\{J_{q-k ; k}(z) E_{k ; \beta} z^{q-k}\right\}=\frac{z^{\frac{\alpha+q}{k}}}{2^{\frac{q}{k}-1}} \sum_{n=0}^{\infty} \frac{z^{(q-k) n}}{\Gamma_{k}(\beta n+k)} \Psi_{2}^{k}\left[\begin{array}{c|c}
(-\alpha+(k-q)(1+k n),-2 k) & z^{2} \\
((k-q)(1+k n),-2 k)(q, k) & \frac{1}{4}
\end{array}\right] .
$$

\section{Conclusion}

In this article, we discuss the product of multi-index Mittag Leffler k-function with Bessel k-function of first kind in fractional integral k-operators, and all results are developed in the form of Wright k-functions. This article is devoted to further research in the field of fractional calculus and special functions. 


\section{Acknowledgment}

The authors would like to thank the anonymous referee for his/her comments, which helped us improve this paper. The research work of Shahid Mubeen is supported by the Higher Education Commission of Pakistan under NRPU Project 2017. All authors read and approved the final manuscript.

\section{References}

[1] R. P. Agarwal, A propos d'une note de M. Pierre Humbert, CR Acad. Sci. Paris, 236 (1953), 2031-2032. 1

[2] A. O. Akdemir, A. Ekinci, E. Set, Conformable Fractional Integrals And Related New Integral Inequalities, J. Nonlinear Convex Anal., 18 (2017), 661-674. 1

[3] M. H. Annaby, Z. S. Mansour, q-fractional Calculus and Equations, Springer, Heidelberg, (2012). 1

[4] Á. Baricz, Generalized Bessel functions of the first kind, Springer-Verlag, Berlin, (2010). 1

[5] R. Diaz, E. Pariguan, On hypergeometric functions and pochhammer k-symbol, Divulg. Mat., 15 (2007), 179-192. 1

[6] M. A. Dokuyucu, A fractional order alcoholism model via Caputo-Fabrizio derivative, AIMS Math., 5 (2020), 781-797. 1

[7] M. A. Dokuyucu, D. Baleanu, E. Celik, Analysis of Keller-Segel model with Atangana-Baleanu fractional derivative, Filomat, 32 (2018), 5633-5643.

[8] M. A. Dokuyucu, E. Celik, H. Bulut, H. M. Baskonus, Cancer treatment model with the Caputo-Fabrizio fractional derivative, Europ. Phys. J. Plus, 133 (2018), 1-6.

[9] M. A. Dokuyucu, H. Dutta, A fractional order model for Ebola Virus with the new Caputo fractional derivative without singular kernel, Chaos Solitons Fractals, 134 (2020), 10 pages. 1

[10] G. A. Dorrego, R. A. Cerutti, The k-Mittag-Leffler function, Int. J. Contemp. Math. Sci., 7 (2012), 705-716. 1

[11] A. Ekinci, M. E. Özdemir, Some New Integral Inequalities Via Riemann-Liouville Integral Operators, Appl. Comput. Math., 18 (2019), 288-295. 1

[12] K. S. Gehlot, J. C. Prajapati, Fractional calculus of generalized k-Wright function, J. Fract. Calc. Appl., 4 (2013), $283-289$. 1

[13] V. Gupta, M. Bhatt, Some results associated with k-hypergeometric functions, Int. J. Appl. Inf. Syst., 5 (2015), 106-109. 1

[14] J. W. Hanneken, D. M. Vaught, B. N. N. Achar, Enumeration of the real zeros of the Mittag-Leffler function $\mathrm{E}_{\mathrm{a}}(z)$, $1<a<2$, In: Advances in Fractional Calculus (Springer, Dordrecht), 2007 (2007), 15-26. 1

[15] A. Kashuri, R. Liko, Ostrowski Type Conformable Fractional Integrals For Generalized (g, s, m, f)-Preinvex Functions, Turkish J. Ineq., 2 (2018), 54-70. 1

[16] M. A. Khan, S. Ahmed, On some properties of the generalized Mittag-Leffler function, SpringerPlus, 2 (2013), 9 pages. 1

[17] A. A. Kilbas, N. Sebastian, Generalized fractional integration of Bessel function of first kind, Integral Transforms Spec. Funct., 19 (2008), 869-883. 1

[18] V. S. Kiryakova, Multiple (multiindex) Mittag-Leffler functions and relations to generalized fractional calculus, J. Comput. Appl. Math., 118 (2000), 241-259. 1

[19] M. Kunt, I. Işcan, Fractional Hermite-Hadamard-Fejér Type Inequalities For GA-Convex Functions, Turkish J. Inequal., 2 (2018), 1-20. 1

[20] G. Mittag-Leffler, Sur la Nouvelle Fonction $\mathrm{E}_{\alpha}(x)$, Comptes Rendus de l'Academie des Sciences Paris, 137 (1903), 554-558. 1

[21] S. R. Mondal, Representation Formulae and Monotonicity of the Generalized k-Bessel Functions, arXiv, 2016 (2016), 12 pages. 1

[22] S. Mubeen, G. M. Habibullah, An integral representation of some k-hypergeometric functions, Int. Math. Forum, 7 (2012), 203-207. 1

[23] D. Nie, S. Rashid, A. O. Akdemir, D. Baleanu, J.-B. Liu, On Some New Weighted Inequalities for Differentiable Exponentially Convex and Exponentially Quasi-Convex Functions with Applications, Mathematics, 7 (2019), 12 pages. 1

[24] M. E. Özdemir, M. Avci-Ardic, H. Kavurmaci-Önalan, Hermite-Hadamard type inequalities for s-convex and s-concave functions via fractional integrals, Turkish J. Sci., 1 (2016), 28-40. 1

[25] A. Petojević, A note about the Pochhammer symbols, Math. Morav., 12 (2008), 37-42. 1

[26] E. D. Rainville, Special functions, Macmillan Co., New York, (1960). 1

[27] T. O. Salim, Some properties relating to the generalized Mittag-Leffler function, Adv. Appl. Math. Anal., 4 (2009), 21-30. 1

[28] T. O. Salim, A. W. Faraj, A generalization of Mittag-Leffler function and integral operator associated with fractional calculus, J. Fract. Calc. Appl., 3 (2012), 1-13.

[29] A. K. Shukla, J. C. Prajapati, On a generalization of Mittag-Leffler function and its Properties, J. Math. Anal. Appl., 336 (2007), 797-811.

[30] H. M. Srivastava, Ż. Tomovski, Fractional calculus with an integral operator containing a generalized Mittag Leffler function in the kernel, Appl. Math. Comput., 211 (2009), 198-210. 1 
[31] D. N. Tumakov, The Faster Methods for Computing Bessel Functions of the First Kind of an Integer Order with Application to Graphic Processors, Lobachevskii J. Math., 40 (2019), 1725-1738. 1

[32] F. B. Yalçin, O Nurgül, Two-Dimensional Operator Harmonically Convex Functions and Related Generalized Inequalities, Turkish J. Sci., 4 (2019), 30-38. 1

[33] H. Yaldiz, A. O. Akdemir, Katugampola Fractional Integrals within the Class of Convex Functions, Turkish J. Sci., 3 (2018), 40-50. 1 